\title{
Refinement of Backward Prediction Method for Reliable Artificial Intelligence-Based Bridge Deterioration Modelling
}

\author{
Jaeho Lee ${ }^{1, *}$, Hong Guan1, Yew-Chaye Loo² and Michael Blumenstein² \\ ${ }^{1}$ The Centre for Infrastructure and Engineering Management (CIEM), Gold Coast Campus, Griffith University, QLD 4222, Australia \\ ${ }^{2}$ Science, Environment, Engineering \& Technology, Griffith University, QLD 4222, Australia
}

\begin{abstract}
A deterioration model is the most critical component of a Bridge Management System (BMS). Artificial Intelligence (AI)-based bridge deterioration model has recently been developed to minimise uncertainties in predicting long-term performance of bridge structural elements. This model contains two components: (1) using Neural Network-based Backward Prediction Model (BPM) to generate unavailable historical condition ratings; and (2) using Time Delay Neural Network (TDNN) to perform long-term performance prediction of bridge structural elements. However new problems have emerged in the process of TDNN prediction. In this study, the BPM-generated condition ratings are used together with the actual overall condition ratings. The incompatibility between the two sets of data produces unreliable prediction outcomes during the TDNN process. This research therefore aims to introduce a new data processing procedure for BPM outcomes, by removing meaningless condition ratings that cause poor training outcomes for long-term prediction using TDNN. Consequently, the outcome of this study can improve accuracy of the current AI-based bridge deterioration model.
\end{abstract}

Key words: bridge deteriorations, bridge management system, artificial neural networks, artificial intelligence.

\section{INTRODUCTION}

Most state and local bridge authorities have become aware of the urgency to develop management technology to ensure safe performance of bridge networks and to facilitate continuous economic growth. Basically, asset management methodologies incorporate economic assessments leading to cost-effective longterm decisions concerning maintenance, repair and rehabilitation (MR\&R). It is particularly important to plan minimum-cost MR\&R activities with funding allocations based on reliable Bridge Management System (BMS) outcomes. BMSs are already a common tool for many bridge authorities to extend the life of bridge networks. However, the reliability of current BMS outcomes is doubtful.
Bridge Deterioration is a stochastic process and the causes of deteriorations are due to diverse explanatory variables including "non-bridge factors" such as traffic intensity, environmental factors, climates etc (Mauch and Madanat 2001; Madanat and Ibrahim 1995). Collection of such variables is not included in current bridge inspection routines. Because of this and with only limited bridge condition rating records, it is very difficult to use typical stochastic-based deterioration models to accurately predict future condition ratings. This limitation has been recognised internationally and it is a challenging problem that has not yet been addressed (Agrawal et al. 2006; DeStefano and Grivas 1998; Madanat et al. 1997; Morcous et al. 2000). A deterioration model is the most critical component of a BMS. The deterioration rate is 
calculated based on historical condition ratings obtained from the structural element-level bridge inspections (Godar and Vassie 1999); it is used to provide information about future bridge deficiencies. Although most bridge authorities have previously conducted inspection and maintenance tasks, these past inspection records are incompatible with what are required by a typical BMS as input. Such incompatibility is a major cause for the deficiency of the current BMS outcomes. Because of this sheer lack of usable inspection records i.e. insufficient historical condition ratings, the outcomes of all current BMSs which incorporate a deterioration model of one type or another are inadequate and unreliable (Lee et al. 2008). For example, the China Bridge Management System (CBMS) has shown, in its early stage of operation, that the reliability of BMS outcomes remains questionable (Yan and Shao 2008). Any BMS should provide effective evaluation and prediction of future bridge performance to aid optimum MR\&R expenditure decisions. Due to the lack of historical inspection data and the weakness of using the weighted overall bridge condition rating method, subjective and qualitative analyses are therefore required for rating, sorting, and planning of future bridge needs. Moreover, the essential deterioration curves, statistic assessment and optimisation techniques are not included in the current CBMS. In other words, the CBMS currently functions more like a Bridge Information System (BIS). This is not the only issue for China. Due to the lack of historical condition rating information, many of bridge authorities worldwide have similar problems in using BMS for accurate and reliable prediction of long-term bridge performance and budget planning.

Further limitation with the current deterioration model is related to the Overall Condition Rating (OCR) methodology used in most existing bridge management technologies. At present, the condition rating information is collected via a quantitative bridge inspection procedure; it is then converted into OCR in a subjective manner. The conditions of bridge elements collected using the element-level bridge inspection process, are expressed quantitatively via the conventional "grading" system, i.e. the health index or the four condition states (CSs 1 to 4). The overall condition of one or more element types of a bridge is calculated with the aid of a weighted average condition state (CS) numbering system. Thus the OCR is incapable of capturing the condition status of individual structural members (i.e. individual beams, piers etc), be it at CS1 (i.e. condition as new or "good"), CS2 ("fair"), CS3 ("poor") or the perilous CS4 ("very poor"). This is a key drawback because bridge collapse usually occurs as a result of the failure of single member(s). In view of this, each of the four CSs for individual members needs to be evaluated in order to reduce the risks of total bridge failure. A further drawback with this stepwise "grading" system is that there are only four CSs with graduation of $1 / 4$ or $25 \%$. Such a step is too large to be used effectively in deterioration modelling. For example, for a CS2 rating the numerical weighting is $62.5 \%$ which is the average of 75 and $50 \%$, whereas in reality the condition of the member can be anywhere between "as new" and "defective". This indeterminacy seriously increases the degree of uncertainty with time in predicting long-term bridge element performance. Note also that it is too expensive to change the current inspection method, which has been used for many years and already produced massive amounts of historical condition rating records. Any change to the inspection method will also create data-incompatibility issues.

In order to address these problems, a study has been conducted recently in an attempt to generate unavailable bridge condition ratings (Lee at el. 2008). This is referred to as the Artificial Intelligence (AI)-based Backward Prediction Model (BPM), which provides unknown historical bridge deterioration patterns to assist in predicting reliable long-term bridge deteriorations. Comparisons with typical bridge deterioration models have also been conducted demonstrating that the prediction errors decrease when the amount of input data increases (Son et al. 2009). This provides further confirmation for the effectiveness of the BPM. In additional to the above, a study has also been conducted using AI-based bridge deterioration modelling technique incorporating the BPM to improve accuracy of long-term prediction (Lee et al. 2008; Son et al. 2010). However, a fundamental shortcoming is discovered in predicting long-term performance of bridge elements due to the use of weighted overall condition rating method. This causes mismatch with the BPM-generated condition ratings which in turn leads to inaccurate long-term predictions. This paper is thus aimed at developing a new analysis process to the existing BPM-based deterioration method thereby minimising the abovementioned problem.

\section{OUTLINE OF THE STUDY}

The BPM methodology in conjunction with the Time Delay Neural Networks (TDNNs) technique has been employed to overcome the fundamental shortcomings mentioned above (Son et al. 2010). A two-stage procedure for the proposed AI-based deterioration model is presented in Figure 1. Also detailed in the figure are indications of the time periods: available condition ratings $\left(t_{p}-t_{p n}\right)$; BPM inputs $\left(t_{0}, t_{p}-t_{p n}\right)$; BPM outputs $\left(t_{1}-t_{p-1}\right)$; BPM and TDNN validation using BPM 


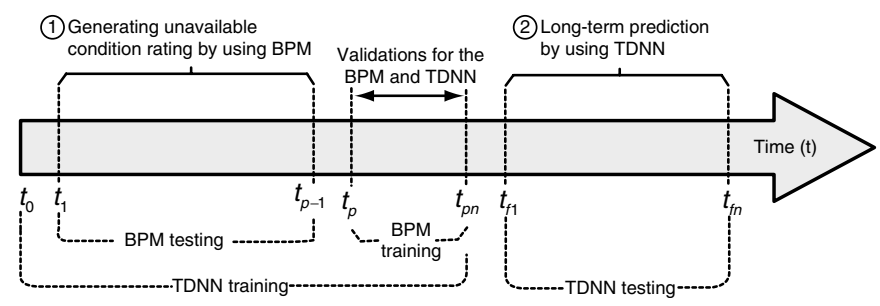

Figure 1. The proposed AI-based bridge deterioration model

outputs $\left(t_{1}-t_{p-1}\right)$; and TDNN long-term prediction $\left(t_{f 1}-t_{f n}\right)$ using condition ratings $\left(t_{0}-t_{p n}\right)$. Note that $t_{0}$ refers to the year of construction completion and its condition rating is assumed to be excellent in this study.

In Stage 1, to generate unavailable historical condition rating $\left(t_{1}-t_{p-1}\right)$, an Artificial Neural Network (ANN)-based BPM is used to establish a correlation between the existing condition rating datasets $\left(t_{p}-t_{p n}\right)$ and the corresponding years' non-bridge factors. The correlations established are then applied to the nonbridge factors $\left(t_{1}-t_{p-1}\right)$ to generate (backward prediction) the missing bridge condition ratings $\left(t_{1}-t_{p-1}\right)$. Therefore, the non-bridge factors, in combination with the ANN technique, can reproduce the historical deterioration trends. The BPM-generated condition ratings for each year contains 66 spectrum rating numbers per CS, which is a combination of learning rates (lr) (0.0-0.5@0.1 increment resulting 6 lr) and momentum coefficients (mc) (0.0-1.0@0.1 increment resulting $11 \mathrm{mc}$ ) in the Neural Network configurations. Note that "mc" is a training parameter that controls how much momentum is used during training session in neural networks. "lr" is also a training parameter and controls the size of weight and bias changes ( $\mathrm{Yu}$ and Liu 2002). The combination of "mc" and "lr", i.e. the total 66 cases in this study represents the total quantity of a given bridge element. For example, if the total quantity of a given bridge element is $100 \mathrm{~m}^{2}$ in area, then each combination of "mc" and " $\mathrm{r}$ " represents $1.52 \mathrm{~m}^{2}$ (i.e. 100/66). The cross-validation is utilised to confirm the reliability of the BPM results. The BPM produces forward prediction $\left(t_{p}-t_{p n}\right)$ using BPM-generated outcomes $\left(t_{1}-t_{p-1}\right)$ only as input values. The results of the forward predictions are then compared with the actual BMS condition ratings $\left(t_{p}-t_{p n}\right)$. Once validated, the BPM backward prediction $\left(t_{1}-t_{p-1}\right)$ together with its forward prediction $\left(t_{p}-t_{p n}\right)$ are eligible to be used as input values for long-term predictions to be carried out in Stage 2.

However when the BPM backward prediction $\left(t_{1}-t_{p-1}\right)$ and its forward prediction $\left(t_{p}-t_{p n}\right)$ are used together as TDNN input, some of the BPM predicted condition ratings are found to be slightly higher than those of the preceding years. Bridge deterioration is a stochastic process and may progress continuously, gradually and slowly (Mauch and Madanat 2001). Thus, the condition rating should not be improved if no MR\&R, i.e. preservation, is undertaken. In other words, this study considers "Do-nothing" - no maintenance effects in long-term prediction. The higher predicted condition ratings cause confusion during the TDNN training session which in turn can yield less-accurate predictions during TDNN testing. To minimise this problem, all BPM-generated condition ratings are required to undergo a filtering process for further improvement of long-term prediction quality. This process is a new addition to the originally developed AIbased deterioration model. The filtering process follows a simple criterion, i.e. Condition Rating (year $n-1) \geq$ Condition Rating $($ year $\mathrm{n}$ ).

In Stage 2, the outcomes of filtered data $\left(t_{1}-t_{p n}\right)$ from Stage 1 and assumed condition rating of the initial year $\left(t_{0}\right)$ are used as TDNN input to predict long-term bridge element performance. The input data is converted to time sequences for time series prediction in the TDNN. The TDNN provides only one-step ahead prediction at a time (one cycle), i.e. 1-year interval in the actual time domain. The result of the first one-stepahead prediction is added onto the original TDNN input $\left(t_{0}-t_{p n}\right)$. This indicates that the number of inputs to the TDNN increases in the second cycle of the one-stepahead prediction. Iterations of the above-described process are required until prediction up to year $t_{f n}$ is completed. The number of yearly prediction by TDNN is also 66, which is in an identical form as the BPM outcomes obtained in Stage 1. Cross-validation is also required to evaluate prediction accuracy of the TDNN outcomes. This is done by comparing the results of TDNN predictions $\left(t_{p}-t_{p n}\right)$ to the known or "actual" BMS condition ratings $\left(t_{p}-t_{p n}\right)$. All TDNN outcomes are considered acceptable if the comparisons are within the same Condition State of the original inspection records. It should be noted that the final results of the TDNN, upon calibration, are also in the same form as the bridge element-level inspection.

\section{IMPROVEMENT OF BPM-BASED BRIDGE DETERIORATION MODEL}

This study is conducted using sample bridge condition rating datasets (i.e. Reinforced Concrete Pier Cap) provided by the Maryland Department of Transport (DoT), USA. Based on the approximate bridge locations provided by the Maryland DoT, non-bridge factors including 4 factors related to the number of vehicles, 2 to the population growth and 15 to climate have been obtained from the Federal Highway Administration, U.S. 
Census Bureau, and the U.S. Department of Commerce National Oceanic and Atmospheric Administration (Lee et al. 2008).

Figure 2 describes the timeframe of input and output for the proposed deterioration model. Only three sets of actual condition rating records (from years 1996 to 2000 with a 2-year increment) are available for both BPM and TDNN. The timeframe shows: (a) available condition ratings; (b) BPM inputs; (c) BPM-generated historical condition ratings; (d) BPM validation inputs; (e) BPM validation outputs; (f) TDNN validation inputs; (g) TDNN validation outputs; (h) TDNN inputs; and (i) TDNN outputs for long-term prediction of bridge elements. Detailed in the following sub-sections are the study outcomes for Stages 1 and 2.

\subsection{Stage 1: Backward Prediction Model (BPM)}

BMS condition rating records obtained from the Maryland DoT is related to a superstructure element Reinforced Concrete Pier Cap (Element \#234 on Bridge \#0301xxxx1). The actual BMS condition rating inputs are collected to periodically update the BMS database. The Condition States (CSs) of BMS condition ratings is 5 grade condition states (CSs) and scaled between CS1 (excellent) and 5 (fail) for the bridge element. The

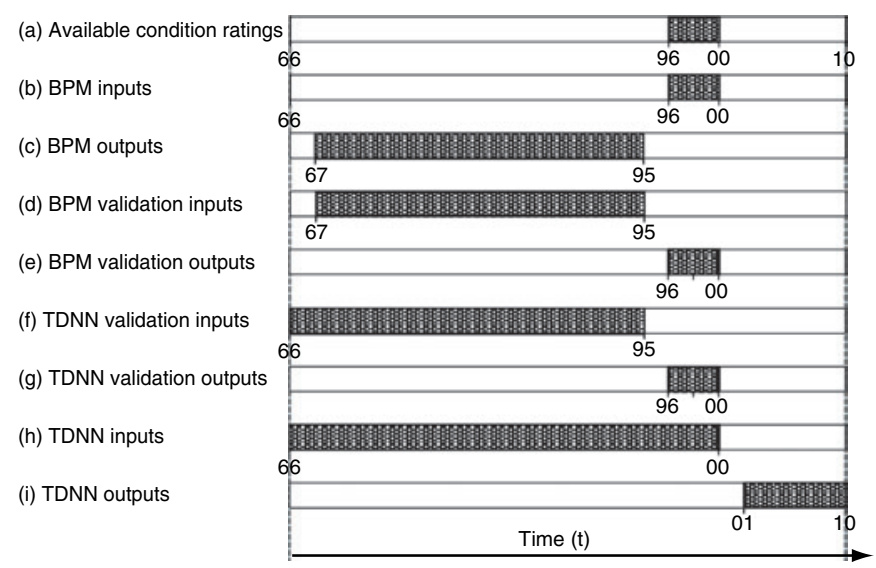

Figure 2. Timeframe of testing the proposed deterioration model (Element \#234 in Bridge \#0301xxxx1) original condition rating information is detailed in Table 1.

The specifications for the inputs, outputs and functions of the BPM are detailed in Table 2. The input layer may have such variables as the number of vehicles, population growth and climatic conditions and more.

\subsubsection{Generating historical condition ratings}

The average quantity of each CS between 1996 and 2000 is about $80 \%, 14 \%$ and $6 \%$ of the total element quantities in CS1, CS2 and CS3, respectively. Figure 3 demonstrates that the BPM generates historical condition ratings from 1967 to 1995 (i.e. 29 years) in three different proportions of the element quantity using the existing condition ratings (1996 to 2000 , i.e. 5 years) together with the assumed condition rating (CS1Excellent) at initial year $\left(t_{0}\right.$, i.e. 1966). Note that in Figure 3 and subsequent similar figures (i.e. Figures 4, 6 and 7) where prediction results are presented, the number of outcomes in each year is 66 which is the combined number of learning rate (lr: 0.0-0.5) and momentum coefficient (mc: $0.0-1.0$ ) in the neural network configuration.

It is noted that the ratio of period of missing/available condition ratings $=29 / 34$ and the ratio of period of available condition ratings/entire period of bridge life $=$ $5 / 34$. This is equivalent to $85.3 \%$ of BPM generated missing years' condition ratings using only $14.7 \%$ of the available condition ratings during the entire period of bridge life.

\subsubsection{Cross validation of BPM-generated condition ratings}

To validate the BPM outcomes, only generated historical condition ratings (years 1967 to 1995) are used as input for forward-prediction of the present years (years 1996 to 2000). Figure 4 shows prediction results (years 1996 to 2000) for three different proportions of the element quantity, i.e. $80 \%, 16 \%$ and $4 \%$ of total element quantity. Note that only 3 years' condition ratings (years 1996, 1998 and 2000) can be used to

Table 1. Raw data of actual condition ratings (Element \#234 on Bridge \#0301xxxx1)

\begin{tabular}{|c|c|c|c|c|c|c|}
\hline \multirow[b]{2}{*}{ Year of inspection } & \multicolumn{6}{|c|}{ Quantity (Each, \%) } \\
\hline & Total & CS1 & CS2 & CS3 & CS4 & CS5 \\
\hline 1996 & $350(100)$ & $280(80)$ & $50(14)$ & $20(6)$ & 0 & 0 \\
\hline 1998 & $350(100)$ & $280(80)$ & $50(14)$ & $20(6)$ & 0 & 0 \\
\hline 2000 & $350(100)$ & $280(80)$ & $50(14)$ & $20(6)$ & 0 & 0 \\
\hline Average (\%) & 100 & 80 & 14 & 6 & 0 & 0 \\
\hline
\end{tabular}




\section{Table 2. Components of the BPM}

\begin{tabular}{|c|c|}
\hline Training Algorithm & Back Propagation Algorithm \\
\hline Transfer Function & Log-sigmoid Function \\
\hline Inputs & $\begin{array}{l}\text { Traffic volume ( } 4 \text { factors), } \\
\text { Population growth } \\
\text { ( } 2 \text { factors) and Climates } \\
\text { (15 factors) }\end{array}$ \\
\hline Total number of input neurons & 21@each year \\
\hline Hidden layers & $\begin{array}{l}2 \text { (15 neurons in } 1 \text { st layer } \\
\text { and } 4 \text { neurons in } 2 \text { nd layer) }\end{array}$ \\
\hline Output & $\begin{array}{l}\text { Bridge Condition Ratings } \\
\text { (1 output@each year) }\end{array}$ \\
\hline Total number of output neurons & 1@each year \\
\hline Scale of learning rate (lr) & $\begin{array}{l}0.0-0.5 \text { in } 0.1 \text { steps } \\
(5 \text { cases })\end{array}$ \\
\hline Scale of Momentum coefficient (mc) & $\begin{array}{l}0.0-1.0 \text { in } 0.1 \text { steps } \\
(11 \text { cases })\end{array}$ \\
\hline Total number of cases generated & $\begin{array}{l}66 \text { (combination of lr and } \\
\text { mc)@each year }\end{array}$ \\
\hline
\end{tabular}

conduct cross-validation. In this study, the maximum prediction error allowance is defined as $\pm 10 \%$. This is half magnitude of a Condition State for a 5 CS-scale.

In Figure 4(c), the predicted minimum condition rating in year 2000 is $35.8 \%$. This value, compared to the lower bound of CS3 (40\% to 60\%), results in a $4.2 \%$ prediction error $\left(\mathrm{CS}_{\min }\right.$ - Predicted condition rating $=$ $40 \%$ to $35.8 \%$ ). This is within the error allowance. Therefore, cross-validation of BPM-generated condition rating is considered satisfactory.

Upon calibration, the BPM-generated condition ratings (i.e. in an identical form as inspection records) are then directly compared to the element quantity with the existing condition information. The prediction error refers to the difference between the BPM-generated condition ratings and the actual element-level condition ratings. If the predictions are slightly over/underestimated for elements in CS1, 2 and 3, the failure risks are minimal because major maintenance and repair work is not required in high condition states. On the other hand, if the predictions are under-estimated for elements in CS4 and 5, they are considered unsatisfactory and unsafe.

Table 3 shows the prediction errors for all five CSs. The total yearly prediction errors are $0.58 \%$ (about 2 out of 350 total quantities), $0.58 \%$ (about 2 out of 350 total quantities), and $4.62 \%$ (about 16 out of 350 total quantities) in years 1996, 1998 and 2000 respectively. Some elements in CS2 and 3 are slightly over/underestimated during this period. However, no elements in CS4 and 5 are under-estimated in this cross-validation. This suggests that the BPM-generated historical condition ratings can be used with confidence as input data in Stage 2.

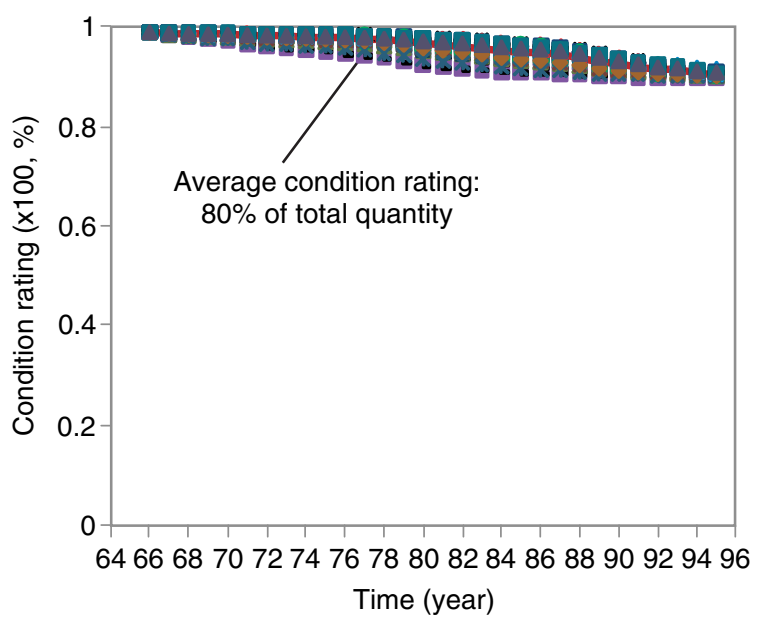

(a) $80 \%$ of total element quantity

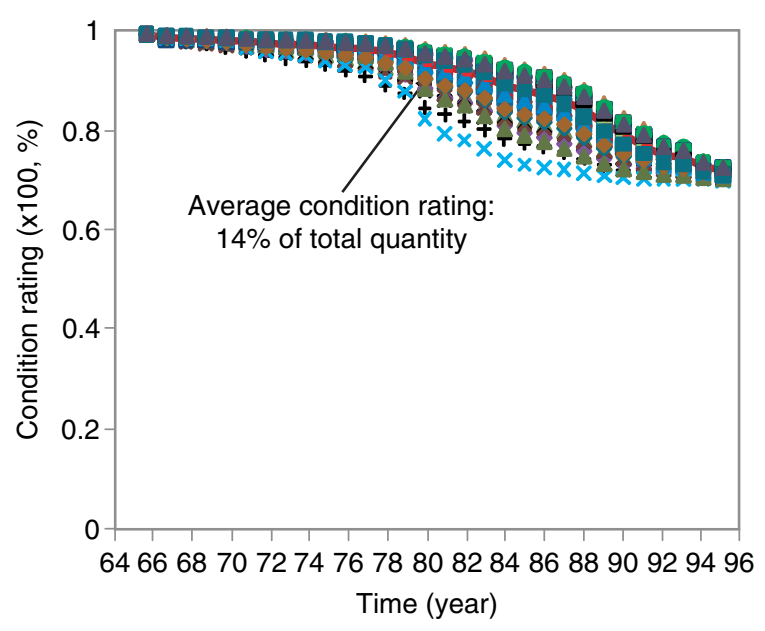

(b) $14 \%$ of total element quantity

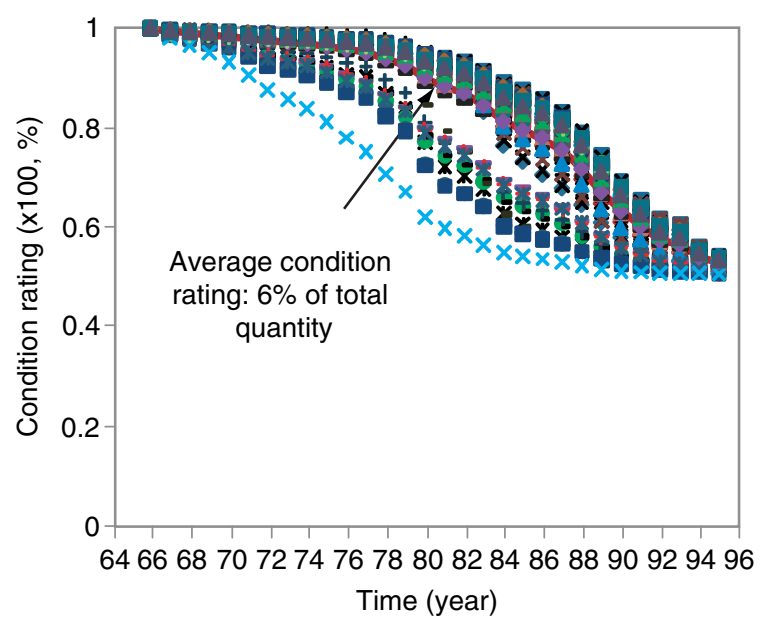

(c) $6 \%$ of total element quantity

Figure 3. BPM results

As mentioned earlier in Section 2, some of the BPM predicted condition ratings are found to be slightly higher than those of the preceding years. This does not 


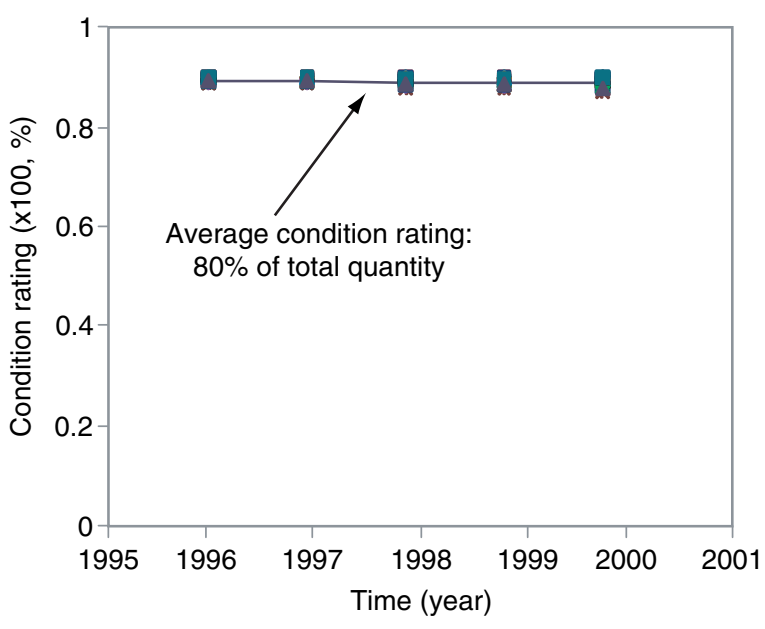

(a) $80 \%$ of total element quantity

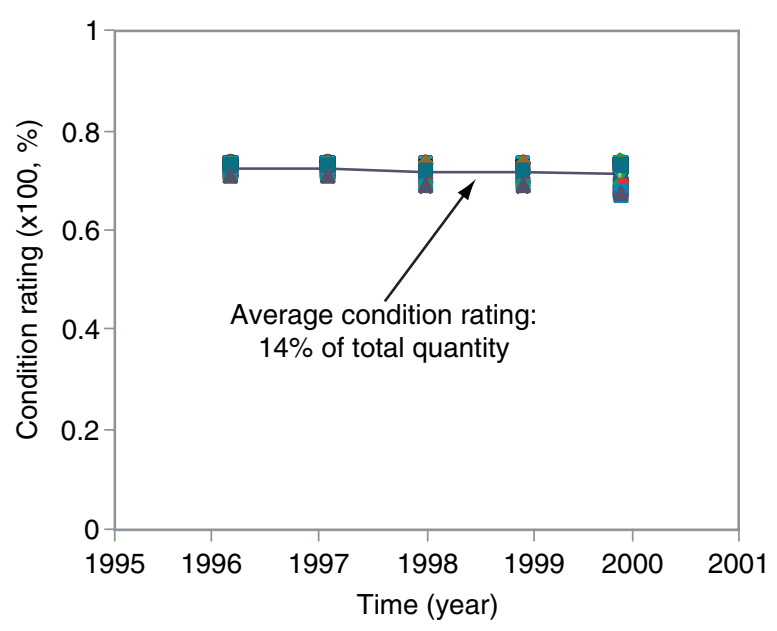

(b) $14 \%$ of total element quantity

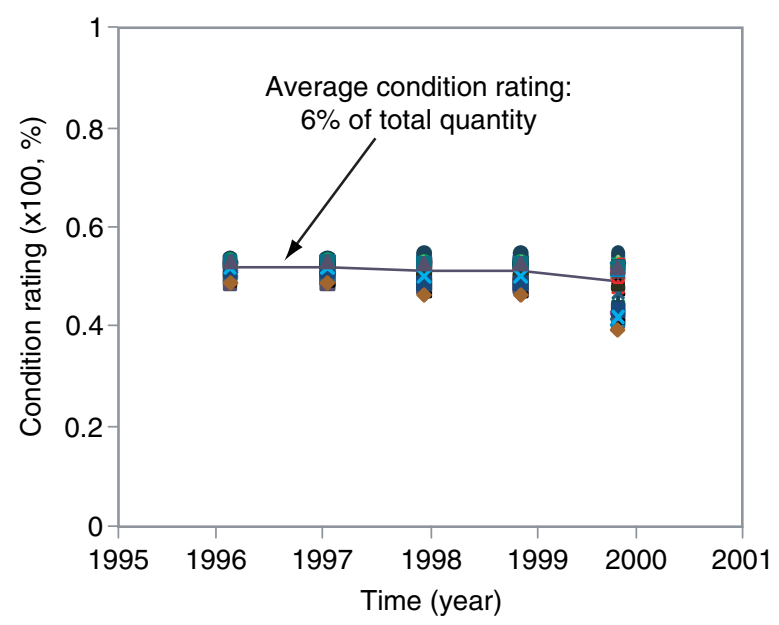

(c) $6 \%$ of total element quantity

Figure 4. BPM results for cross-validation (1996, 1998 and 2000)

Table 3. Prediction errors of the BPM using forward comparisons (Bridge \#0301xxxx1)

\begin{tabular}{|c|c|c|c|c|c|c|c|}
\hline \multirow[b]{2}{*}{ Year } & & \multicolumn{6}{|c|}{ Element quantity (\%) } \\
\hline & & CS1 & CS2 & CS3 & CS4 & CS5 & Total \\
\hline \multirow[t]{3}{*}{1996} & A & 80.00 & 14.29 & 5.71 & 0.00 & 0.00 & 100 \\
\hline & B & 80.00 & 14.00 & 6.00 & 0.00 & 0.00 & 100 \\
\hline & $\mathrm{C}$ & 0.00 & 0.29 & 0.29 & 0.00 & 0.00 & 0.58 \\
\hline \multirow[t]{3}{*}{1998} & A & 80.00 & 14.29 & 5.71 & 0.00 & 0.00 & 100 \\
\hline & B & 80.00 & 14.00 & 6.00 & 0.00 & 0.00 & 100 \\
\hline & $\mathrm{C}$ & 0.00 & 0.29 & 0.29 & 0.00 & 0.00 & 0.58 \\
\hline \multirow[t]{3}{*}{2000} & A & 80.00 & 11.69 & 7.79 & 0.52 & 0.00 & 100 \\
\hline & B & 80.00 & 14.00 & 6.00 & 0.00 & 0.00 & 100 \\
\hline & $\mathrm{C}$ & 0.00 & 2.31 & 1.79 & 0.52 & 0.00 & 4.62 \\
\hline
\end{tabular}

A:Results, B: Existing records, C: Difference of A and B

match with the absence of MR\&R activities. The total number of yearly prediction by TDNN is 198 , as a result of 66 cases for each of the three proportions $(80 \%, 14 \%$,
$6 \%$ of total element quantity). It is observed that 88 cases out of the total 198 cases have their condition ratings predicted unrealistically higher. The jumps in 
condition ratings in three proportions are plotted in Figure 5, where the maximum errors are respectively $0.61 \%, 3.26 \%, 1.14 \%$ in $80 \%, 14 \%, 6 \%$ of the total element quantity. The corresponding average errors are $0.41 \%, 1.49 \%, 0.69 \%$. Through a data filtering process, the BPM-generated condition ratings satisfying the

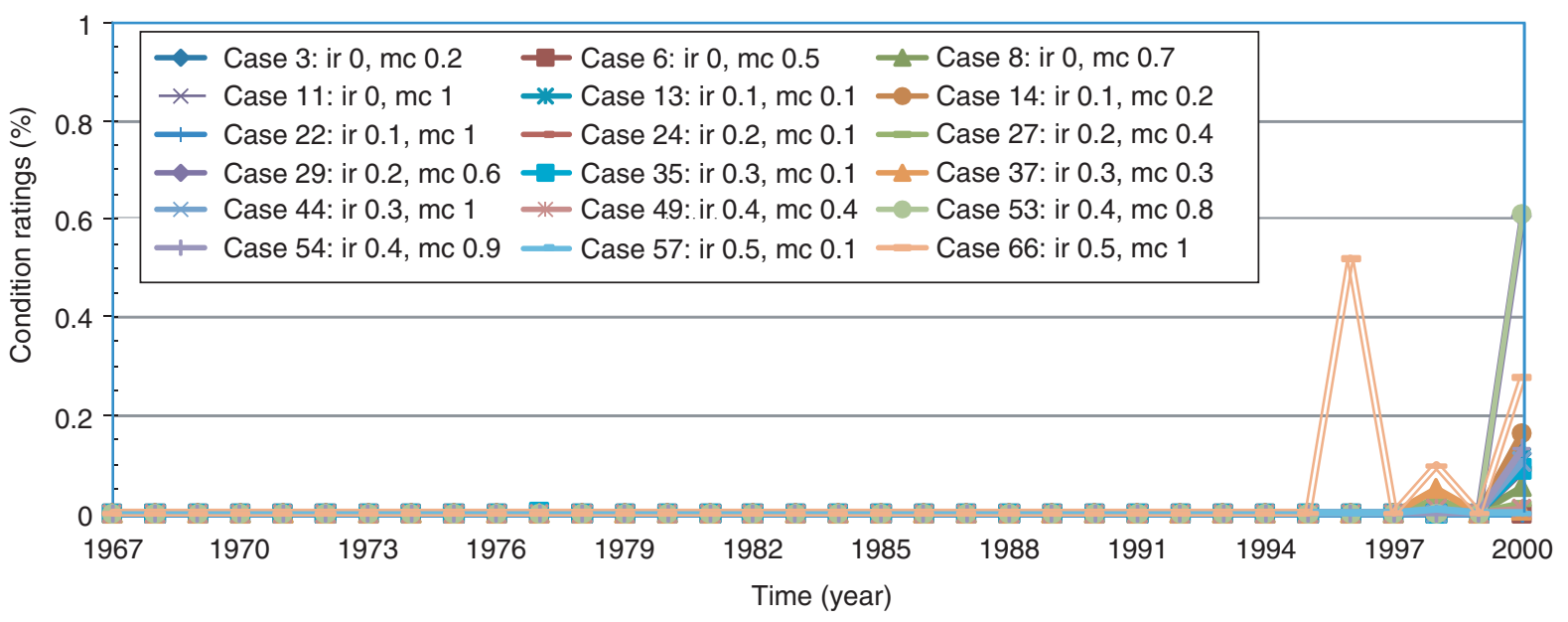

(a) $80 \%$ of total element quantity

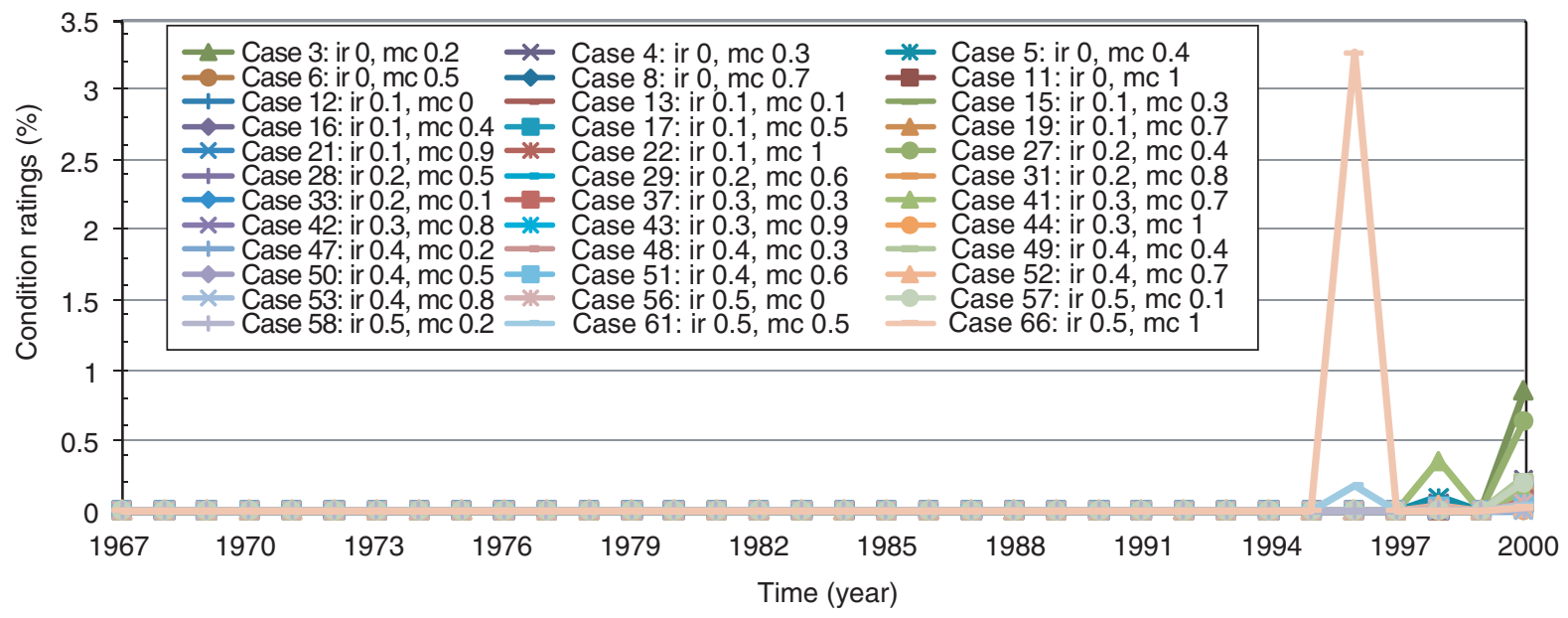

(b) $14 \%$ of total element quantity

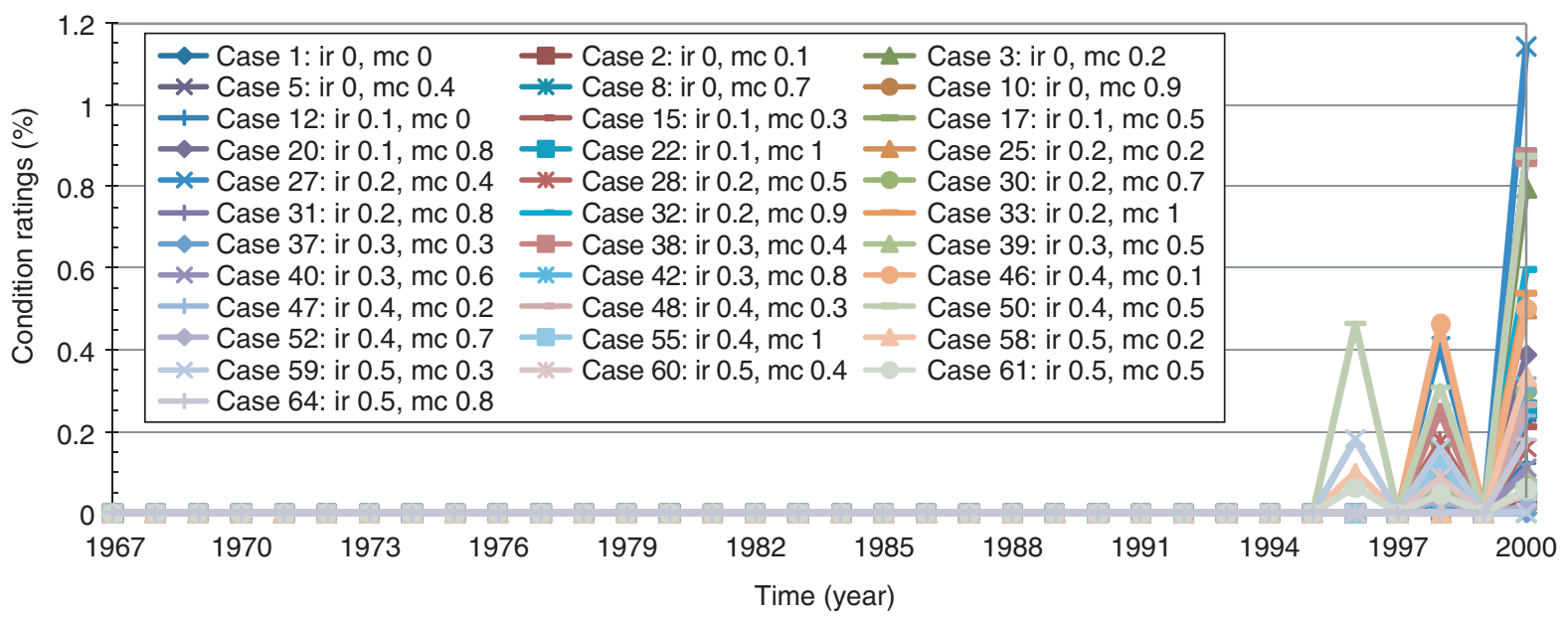

(c) $6 \%$ of total element quantity

Figure 5. Data filtering on the BPM-generated condition ratings 
following criteria, Condition Rating $($ year $n-1) \geq$ Condition Rating $_{(\text {year } n)}$, should be removed and replaced by the identical condition rating of preceding year

\subsection{Stage 2: Time Delay Neural Networks (TDNNs)}

Before performing long-term prediction for years 2001-2010, the TDNN outputs are required to be validated. A short-term prediction for years 1996 to 2000 is considered for cross-validation to measure prediction accuracy of the proposed deterioration model.

\subsubsection{A short-term prediction for cross-validation}

The BPM-generated historical condition ratings (years 1967 to 1995) together with the assumed condition rating at the year of construction completion (year 1966) are used as TDNN inputs for the short-term prediction (for years 1996 to 2000). One-step-ahead TDNN prediction is conducted up until year 2000. The outcomes (years 1996 to 2000) of TDNN are subsequently compared with the actual condition rating records (years 1996, 1998, 2000) for further confirmation of BPM-generated data. The TDNN short-term prediction, as cross-validation, is presented in Figure 6. It is found in Figure 6(c) that the minimum condition rating predicted for CS3 in year 2000 is $38.75 \%$. When compared to the lower bound of CS3 $(40 \%$ to $60 \%)$, a $1.25 \%\left(\mathrm{CS} 3_{\min }\right.$ - predicted condition rating $=40 \%$ to $38.75 \%$ ) prediction error is produced which is within the error allowance $( \pm 10 \%)$. Therefore, cross-validation for TDNN short-term prediction using BPM-generated condition ratings is also considered satisfactory.

A post-calibration process is also required to convert the generated condition ratings to element quantities thereby enabling comparison with the actual

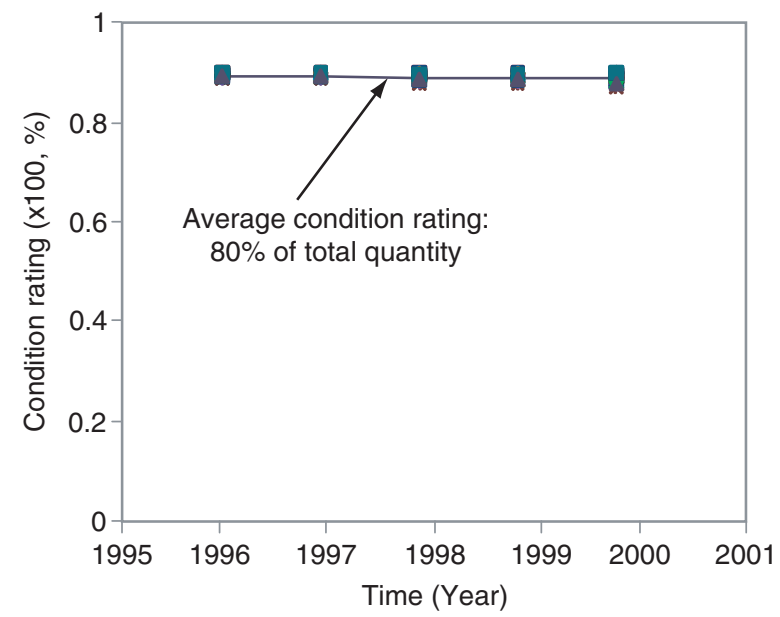

(a) $80 \%$ of total element quantity

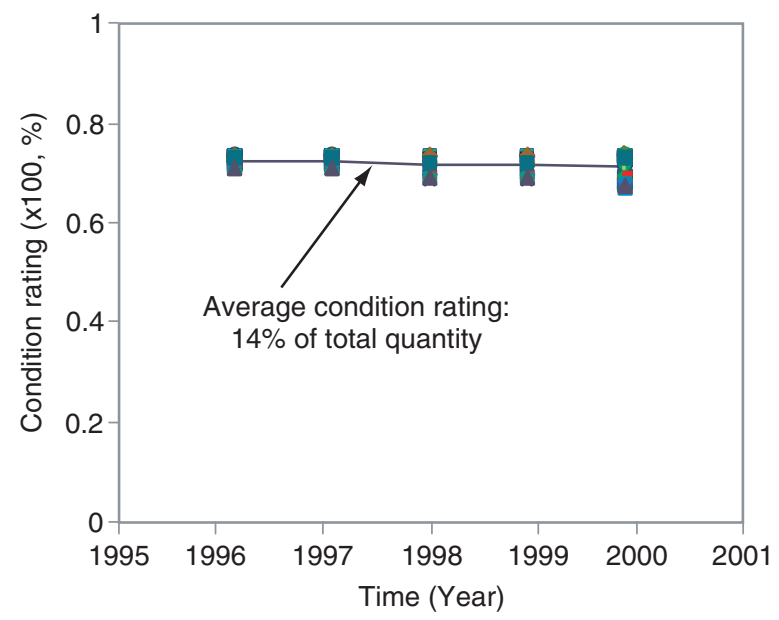

(b) $14 \%$ of total element quantity

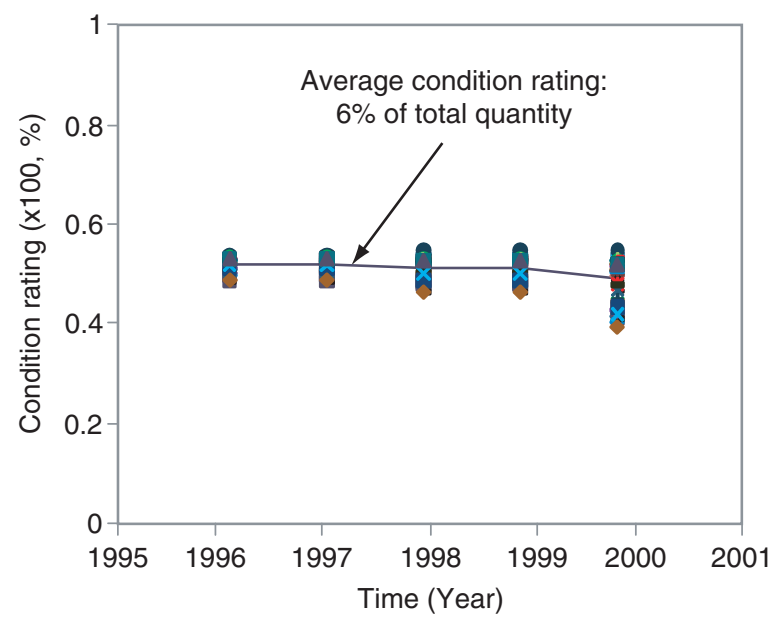

(c) $6 \%$ of total element quantity

Figure 6. TDNN short-term prediction results for cross-validation (years 1996, 1998, and 2000) 
Table 4. Comparison of results

\begin{tabular}{lrrrrrrr}
\hline \multicolumn{7}{c}{ Year } & \multicolumn{7}{c}{ Element quantity (\%) } \\
\cline { 3 - 8 } 1996 & CS1 & CS2 & CS3 & CS4 & CS5 & Total \\
& A & 80.00 & 14.00 & 6.00 & 0.00 & 0.00 & 100 \\
& B & 80.00 & 14.00 & 6.00 & 0.00 & 0.00 & 100 \\
1998 & C & 0.00 & 0.00 & 0.00 & 0.00 & 0.00 & 0.00 \\
& A & 80.00 & 14.00 & 6.00 & 0.00 & 0.00 & 100 \\
& B & 80.00 & 14.00 & 6.00 & 0.00 & 0.00 & 100 \\
2000 & C & 0.00 & 0.00 & 0.00 & 0.00 & 0.00 & 0.00 \\
& A & 80.00 & 14.00 & 5.09 & 0.91 & 0.00 & 100 \\
& B & 80.00 & 14.00 & 6.00 & 0.00 & 0.00 & 100 \\
& C & 0.00 & 0.00 & 0.91 & 0.91 & 0.00 & 0.91 \\
\hline
\end{tabular}

A:Results, B: Existing records, C: Difference of A and B

element quantities. These are presented in Table 4. It can be seen that differences in element quantities exist only in CS3 and 4 in year 2000. CS4 is over-predicted by $0.91 \%$ (i.e. about 3 out of 350 total element quantity). This is because CS3 in the same year is under-predicted by $5.09 \%$. However, no elements in CS4 and 5 are under-estimated in this cross-validation. This means that the all BPM-generated historical data can be used as input for the TDNN long-term prediction.

\subsubsection{TDNN long-term prediction}

Once validated, all historical condition ratings, i.e. assumed condition rating at year of construction completion (year 1966, together with the BPMgenerated missing condition ratings (years 1967 to 2000), are used as TDNN inputs. The result of the first one-step-ahead prediction (i.e. year 2001) is added on to the original TDNN input (years 1967 to 2000). Iterations of this one-step-ahead prediction process are continued until year 2010 for a 10-year long-term prediction. Figure 7 shows the results of long-term prediction of condition ratings (years 2001 to 2010) for bridge element \#234: Figure 7(a) $80 \%$ of total element quantities; Figure 7(b) 14\% of total element quantities; Figure 7 (c) $6 \%$ of total element quantities. These prediction results have assumed no maintenance effect.

Figure 8 shows a summary of the predicted condition states in quantity form and their annual overall condition ratings for the 10-year long-term performance (years 2001 to 2010) using yearly BPM-generated condition ratings (years 1967 to 2000). The advantage of the proposed deterioration model is that it can provide quantity of each CS thereby overcoming the shortcomings of OCR which ignores critical failure risks in small number of bridge elements with low Condition States (CSs).

\section{DISCUSSION AND SUMMARY}

The previously developed AI-based bridge deterioration modelling technique incorporating the Backward Prediction Model (BPM) (Son et al. 2010) has proven to be able to improve accuracy of long-term prediction. The current model, however, is computationally less efficient due to the time consuming Neural Network training process, which generates a huge amount of data. The BPM-generated condition ratings for each year contain 66 spectra of rating numbers per CS, which is a combination of the learning rates (lr) (i.e. lr: 0.0 to 0.5 @ 0.1 increment, resulting in $6 \mathrm{lr}$ ), and momentum coefficients (mc) (mc:0.0 to 1.0 @ 0.1 increment resulting in $11 \mathrm{mc}$ ) in the Neural Network configurations. The number 66 (i.e. $6 \times 11$ ) applies to given element type. The 66 BPM outcomes per CS per year create a huge number of combined scenarios in long-term prediction stage; for four CSs in $\mathrm{n}$ years it gives $(66 \times 4)^{\mathrm{n}}$. As the initial settings prior to Neural Network training are a result of a random data process, many of the combined scenarios are less meaningful which need to be filtered out to further increase prediction accuracy and efficiency. To achieve this ultimate goal, this study presents a data filtering process as a first step, by which the BPMgenerated condition ratings can provide logical information for reliable long-term prediction of bridge elements by TDNN. The TDNN is one of the sensitive and reliable dynamic analysis techniques for signal processes and speech recognitions, because it has a strong ability to detect patterns of dynamic input data. However, irregular noise pattern(s) or illogical pattern(s) leads poor training results.

The first stage of the AI-based deterioration model is to generate unavailable condition ratings. Based on 3 sets of available condition ratings (or $14.7 \%$ of the total period), the BPM is able to generate 29 missing condition ratings (or $85.3 \%$ of the total period) when 


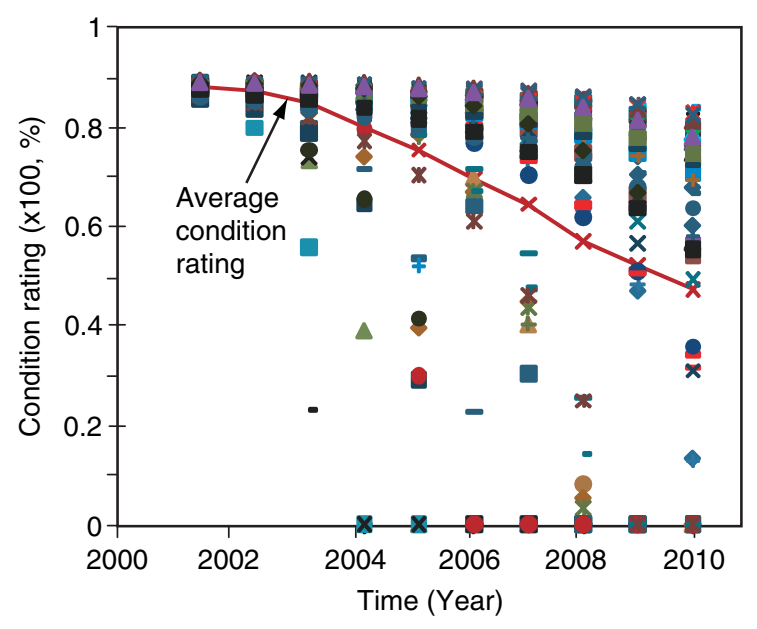

(a) $80 \%$ of total element quantity

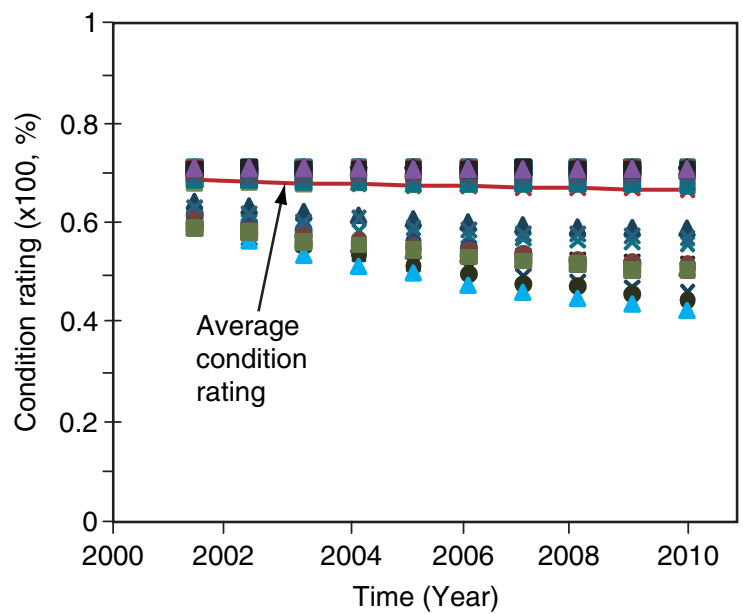

(b) $14 \%$ of total element quantity

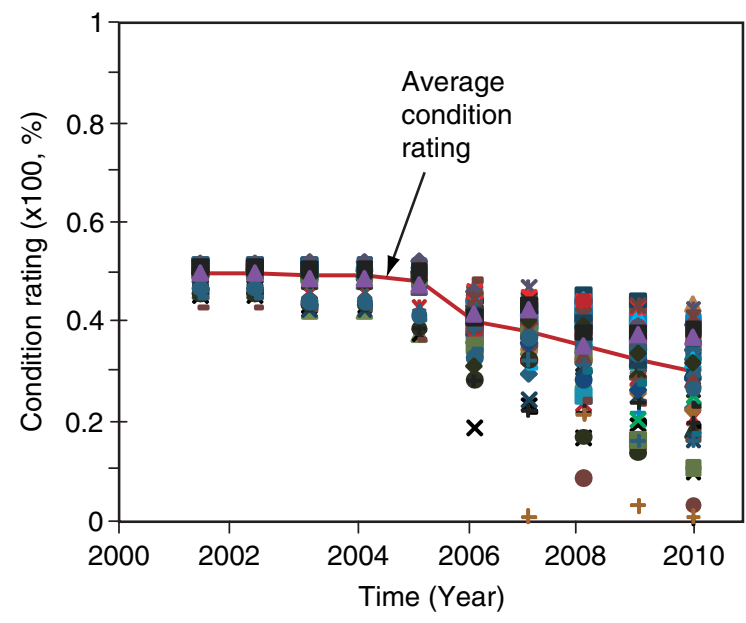

(c) $6 \%$ of total element quantity

Figure 7. TDNN long-term predictions - "Do Nothing" maintenance effect

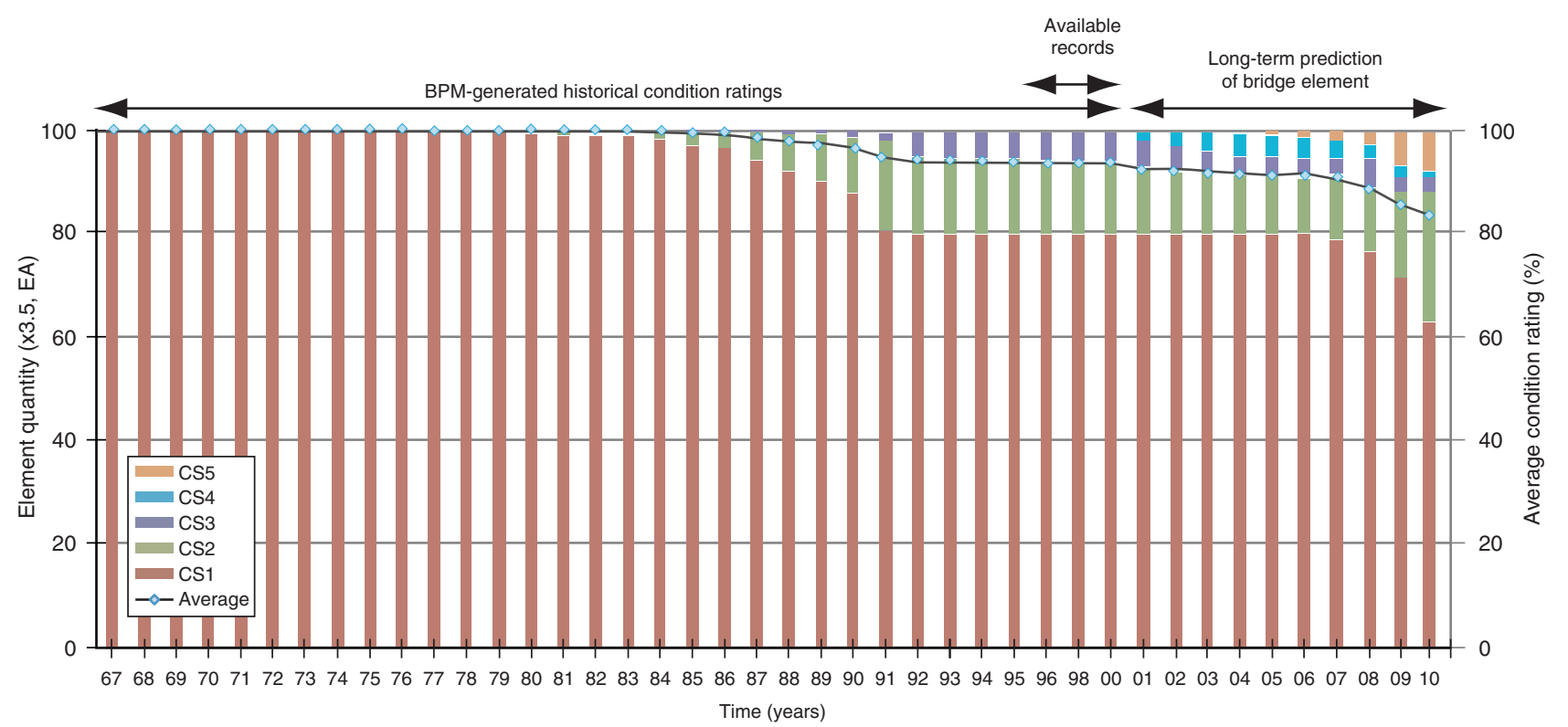

Figure 8. Summary of predicted condition states 
proper inspection records were missing. The prediction error of the condition rating in year 2000 is $4.2 \%$, which is within the error allowance $( \pm 10 \%)$. The maximum total yearly prediction error is found to be $4.62 \%$ (about 16 out of 350 total element quantities) in year 2000 .

This stage also involves data filtering process on all BPM-generated condition rating outcomes. The illogically predicted condition ratings are filtered out which is equivalent to $44 \%$ of the predicted data (i.e. 88 cases out of 198 cases). The resulting maximum and average maximum errors are $3.26 \%$ and $1.49 \%$, respectively.

During the second stage, long-term prediction of bridge element condition ratings is performed using TDNN technique. In this study, the TDNN process considers "no maintenance" effects only. A short-term prediction for cross-validation is performed to reconfirm the prediction accuracy of BPM-generated missing condition ratings and the reliability of TDNN. The results of short-term prediction (years 1996 to 2000) are compared with the existing condition ratings for the same time period (years 1996 to 2000). Some results are over-estimated by $1.25 \%$ in CS3 in year 2000. However, this prediction error is within the error allowance. Therefore, it can be ascertained that all BPM-generated condition ratings can be used as input for TDNN long-term prediction.

Upon validation, full historical condition ratings including assumed condition rating at year of construction completion (year 1966), BPM-generated historical condition ratings (years 1967 to 1995), and the existing condition ratings (years 1996 to 2000) can be used for TDNN long-term prediction (10 years in this study). As seen in Figure 8, the outcomes of TDNN predictions are in the same form as quantitative condition rating inspections. This suggests that the predictions are more descriptive than the typical OCR method in terms of elements quantities in each CS.

\section{CONCLUSION}

Recognising the historical patterns for aging bridges can be a problem when using commonly available time series prediction methods. This is therefore a fundamental benefit of using AI-based BPM method for long-term bridge performance prediction. As results demonstrated, the predicted condition ratings can also be expressed in the same form as the actual inspection records. This enables prediction of element quantities for each bridge element. The proposed AI-based deterioration model takes into account critical failure risks in small number of bridge elements in low CSs. This implies that the risk, due to the limitation of the OCR method, can be minimised in long-term predictions. To this end, the present study has established the methodology for reliable long-term prediction of condition ratings of bridge elements using only limited sample data.

Despite the advancement of the current AI-based deterioration model, only short-term bridge element condition ratings are found to be reliably predicted. The longer-term predictions are practically unreasonable, i.e. the deterioration rate increases rapidly, due to some illogically generated condition ratings by the BPM. To overcome this, data filtering process is added on to the current AI-based deterioration model. Higher than preceding year's condition rating values in the generated dataset are removed and replaced by the identical condition rating of the preceding year. The filtering process has helped further improvement of the prediction accuracy. Based on the outcome of the present study, further case studies should be carried out to confirm the methodology and to improve time efficiency in typical ANN training process. Further work should also consider the maintenance effects in predicting long-term bridge element performance. Nevertheless, the outcome of the present study is very useful for further development of a reliable bridge deterioration model for BMSs.

\section{ACKNOWLEDGEMENT}

The resources used for the present study were provided by the Maryland State Department of Transportation, U.S. The authors wish to thank Messrs Earle Freedman and Matt Zulkowski for providing necessary information requested.

\section{REFERENCES}

Agrawal, A., Kawaguchi, A. and Chen, Z. (2010). "Deterioration rates of typical bridge elements in New York", Journal of Bridge Engineering, ASCE, Vol. 15, No. 4, pp. 419-429.

DeStefano, D. and Grivas, A. (1998). "Method for estimating transition probability in bridge deterioration models", Journal of Infrastructure System, Vol. 4, No. 2, pp. 56-62.

Godar, B. and Vassie, R. (1999). Review of Existing BMS and Definition of Inputs for the Proposed BMS, Deliverable D4 BRIME Report, PL97-2220.

Lee, J.H., Sanmugarasa, K., Blumenstein, M. and Loo, Y.C. (2008). "Improving the reliability of a bridge management system (BMS) using an ANN-based backward prediction model (BPM)", Automation in Construction, Vol. 17, No. 6, pp. 758-772.

Mauch, M. and Madanat, S. (2001). "Semiparametric hazard rate models of reinforced concrete bridge deck deterioration", Journal of Infrastructure Systems, Vol. 7, No. 2, pp. 49-57.

Madanat, S. and Ibrahim, W.H.W. (1995). "Poisson regression models of infrastructure transition probabilities", Journal of Transportation Engineering, Vol. 121, No. 3, pp. 267-272. 
Madanat, S.M., Karlaftis, M.G. and McCarthy, P.S. (1997). "Probabilistic infrastructure deterioration models with panel data", Journal of Infrastructure System, Vol. 3, No. 1, pp. 120-125.

Morcous, G., Rivard, H. and Hanna, A. (2000). "Case-based reasoning system for bridge management", Proceedings of the $8^{\text {th }}$ International Conference on Computing in Civil and Building Engineering, Stanford, California, USA, August, pp.1363-1370.

Son, J.B., Lee, J.H., Blumenstein, M., Loo, Y.C., Guan, H. and Panuwatwanich, K. (2009). "Improving reliability of bridge deterioration model using generated missing condition ratings", Proceedings of the $3^{\text {rd }}$ International Conference on Construction Engineering and Management (ICCEM), Jeju, Korea. (CD$\mathrm{ROM})$
Son, J.B., Lee, J.H., Guan, H., Loo, Y.C. and Blumenstein, M. (2010). "ANN-based structural element performance model for reliable bridge asset management", Proceedings of the $21^{\text {st }}$ Australasian Conference on Mechanics of Structures and Materials, Melbourne, Australia, pp. 775-780.

Yan, B.F. and Shao, X.D. (2008). "Application of China bridge management system in Qinyuan city", Bridge Maintenance, Safety, Management, Health Monitoring and Informatics, D.M. Frangopol and H.M. Koh, eds., CRC Press, London, UK.

Yu, C.C. and Liu, B.D. (2002). "A back propagation algorithm with adaptive learning rate and momentum coefficient", Proceedings of the 2002 International Joint Conference on Neural Networks, Honolulu, Hawaii, USA, pp. 1218-1223. 